\title{
Aplicação de recursos fisioterapêuticos no tratamento de estrias
}

\author{
Application of physiotherapeutic resources in the treatment of stretch marks \\ Aplicación de recursos fisioterapéuticos en el tratamiento de estrías
}

Recebido: 30/11/2021 | Revisado: 07/12/2021 | Aceito: 08/12/2021 | Publicado: 17/12/2021

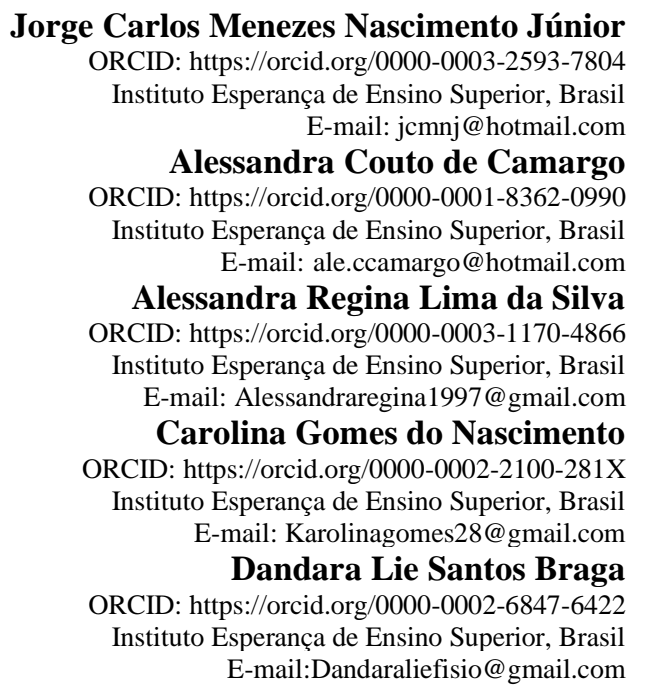

\begin{abstract}
Resumo
Objetivo: Analisar a utilização de recursos fisioterapêuticos, tais como Vacuoterapia e Microgalvanopuntura no tratamento direto de estrias atróficas. Métodos: Trata-se de uma revisão sistemática descritiva do tipo transversal. Embasada em levantamento feito nas plataformas digitais Scielo, Google acadêmico e Capes periódica. Os artigos encontrados foram no idioma da língua portuguesa. O critério de inclusão utilizado foram os artigos que apresentassem estudos de casos com a temática da aplicação dos recursos terapêuticos no tratamento de estrias atróficas. Resultados: De maneira geral os estudos apresentaram resultados promissores quanto ao uso dos recursos terapêuticos em indivíduos que relataram ter estrias atróficas em alguma área corporal.
\end{abstract}

Palavras-chave: Estrias; Vacuoterapia; Microgalvanopuntura; Ventosaterapia.

\begin{abstract}
Objective: To analyze the use of physical therapy resources, such as Vacuum Therapy and Microgalvanopuncture in the direct treatment of atrophic streaks. Methods: This is a descriptive, cross-sectional systematic review. Based on a survey carried out on the digital platforms Scielo, Academic Google and periodic Capes. The articles found were in the Portuguese language. The inclusion criteria used were articles that presented case studies with the theme of the application of therapeutic resources in the treatment of atrophic streaks. Results: In general, the studies showed promising results regarding the use of therapeutic resources in individuals who reported having atrophic streaks in some body area.
\end{abstract}

Keywords: Stretch Marks; Vacuum Therapy; Microgalvanopuncture; Wind therapy.

\section{Resumen}

Objetivo: Analizar el uso de recursos de fisioterapia, como la Terapia de Vacío y la Microgalvanopunción en el tratamiento directo de estrías atróficas. Métodos: Se trata de una revisión sistemática descriptiva y transversal. Basado en una encuesta realizada en las plataformas digitales Scielo, Academic Google y Capes periódicas. Los artículos encontrados estaban en idioma portugués. Los criterios de inclusión utilizados fueron artículos que presentaran estudios de caso con el tema de la aplicación de recursos terapéuticos en el tratamiento de estrías atróficas. Resultados: En general, los estudios arrojaron resultados prometedores en cuanto al uso de recursos terapéuticos en individuos que refirieron tener estrías atróficas en alguna zona del cuerpo.

Palabra clave: Estrías; Vacuumterapia; Microgalvanopuntura; Ventosatherapy. 


\section{Introdução}

O surgimento das estrias no corpo de um indivíduo causa transtornos que impedem a prática de atividade física e lazer por conta da exposição a olho nu da pele, que acarreta em uma baixa autoestima, o que interfere seriamente no psicossocial do indivíduo, principalmente em mulheres que têm fragilidade e sofrem por falta de aceitação do próprio corpo devido às críticas sociais. As mulheres têm a tendência de adquirir transtornos pelo fato de que precisam seguir um padrão de beleza, então quando ocorre a presença dessas estrias nas áreas corporais, gera uma alteração na composição estrutural da pele, levando ao desinteresse na hora de expor o seu corpo no meio social e a perda da confiança em si mesma.

Desta forma as estrias são classificadas como lesões lineares que surgem na pele devido ao mecanismo externo que desencadeia o estiramento tecidual sendo eles: efeito mecânico, uso de próteses mamárias e hormonal. Já no mecanismo interno, ocorre devido marcadores genéticos, o crescimento acelerado e ganho de massa muscular, com incidência em regiões específicas como nádegas, abdômen e mamas. Desta maneira é possível que o tecido epitelial desenvolva uma aparência enrugada, de proporções lineares e paralelas, obedecendo as linhas de clivagem do tecido e tem como principal sintoma o prurido caracterizado por uma coceira na pele. Atualmente, a grande procura de tratamento estético é do grupo de mulheres com objetivo de melhorar a aparência. Estima-se que as estrias acometem 2,5 vezes mais em mulheres do que em homens, elas aparecem por volta dos 12 a 14 anos em adolescentes do sexo feminino (Gerusa, Nitsch, Nascimento, 2017).

Trata-se então de uma atrofia tegumentar adquirida que é ocasionada pela ruptura das fibras elásticas (elastólise), resultado da granulação de mastócitos e ativação macrófica (Guirro, 2002). Podendo variar de acordo com a aparência, no momento inicial surgem as estrias vermelhas/roxas, que possuem essa coloração devido a quantidade de vasos remanescente que ainda irrigam a região e após isso vem as esbranquiçadas, ou seja, ocasionando interrupção da irrigação local que são adquiridas devido uma fibrose (Borges, 2010; Guirro \& Guirro, 2002; Mondo, 2004).

A formação das estrias tem como ponto inicial o aspecto de coloração vermelha no local. Durante esse período de cor vermelha ou rosada, significa que naquela determinada região ainda ocorre o processo inflamatório e as chances de sucesso no tratamento são maiores, isso é possível devido a existência de fibras elásticas.

Uma das principais abordagens que engloba a ventosaterapia é a vacuoterapia, uma antiga terapia usada pelos egípcios, com uso de ventosas mais tarde aprimorada pela medicina chinesa, até chegar aos dias atuais. Consiste em um procedimento não invasivo que por meio da aplicação de uma pressão negativa, induz uma sucção na pele que pode ser prolongada ou de curta duração. A aspiração permanece até que haja um pequeno sangramento, que mais tarde causará uma depressão no local, dando origem a uma prega cutânea e o rolamento de estruturas adjacentes. A hiperestimulação dos vasos sanguíneos promove uma troca gasosa mais abundante entre o tecido e os vasos capilares, além de que devido a pressão exercida na sucção, ocorre um desfibrosamento na região fazendo com que as células de colágeno e elastina recuperem a tonicidade e a textura elástica. (Silva, et al, 2020).

Outra abordagem que também possibilita resultados satisfatório e o tratamento da galvanoterapia que através de auxílio da punturação emitirá um estímulo na proximidade da agulha que causará um processo inflamatório, no qual esse processo ajudará na produção de fibroblastos, fibras elásticas, colágenos e melhora da aparência da estria.

É comum encontrar indivíduos com problemas de autoestima devido ao aparecimento de estrias, que eleva o aumento exponencial da procura pelo corpo ideal. Contudo, durante a pesquisa em artigos científicos não foi comprovado uma cura definitiva para as estrias, sem que ocorresse a retirada do tecido estriado, porém existem métodos de tratamento paliativos como peeling, hidratação da pele, vacuoterapia, laserterapia, microdermoabrasão, escarificação, eletroterapia, uso de medicamentos e massagem que ajudam a minimizar o aspecto enrugado e a coloração visualmente (Gerusa, Nitsch, Nascimento, 2017). 
Esses tratamentos são usados com intuito de promover a redução da espessura das estrias nas áreas corporais dos indivíduos. Dessa forma é aplicado a técnica de vacuoterapia e microgalnopuntura que veio ganhando seu espaço atualmente entre os profissionais da área da saúde, esses tratamentos têm demonstrado grandes resultados em melhorar o aspecto das estrias.

Antigamente o assunto de cuidados com a pele era considerado irrelevante em meio aos indivíduos do sexo masculino, no qual a atitude não passava apenas de um aspecto de vaidade e beleza com relação ao público feminino. Já que as mesmas possuem maior preocupação em cuidar do próprio corpo e de como querem ser vistas pela sociedade. Aos poucos esses tabus foram sendo quebrados e revertidos para que todos pudessem ver a importância e a relevância de adquirir hábitos de cuidados com a pele.

Com o surgimento das estrias, o impacto gerado nas pessoas é inevitável, ao ponto em que não se trata apenas de uma lesão linear, para muitas pessoas isso faz a total diferença, pois essas estrias são capazes de proporcionar mudanças no comportamento físico e psicológico, tanto que muitas pessoas se limitam quanto a maneira de se vestir, evitam locais de lazer que exijam maior exposição do corpo como roupas de banho e outras situações da convivência no meio social.

Assim, essa pesquisa busca evidenciar o uso de abordagens fisioterapêuticas não invasivas conhecidas como ventosaterapia e vacuoterapia. Essas técnicas com o decorrer do tempo, vem ganhando seu espaço no uso clínico, com intuito de proporcionar bem estar ao indivíduo. O seu mecanismo de funcionamento se dá através de uma pressão negativa por meio de uma pistola com encaixe exclusivo para o pino do copo de ventosa. Na vacuoterapia é utilizado uma pressão negativa aplicado na região da estria de maneira não invasiva, será realizado o arrasto até que cause uma sangria. Assim induzindo um processo inflamatório para que haja uma nova produção de substâncias como colágenos e elastina potencializando os efeitos em cima de estrias a fim de reduzir o seu tamanho e aparência.

O objetivo principal deste estudo é analisar os dados coletados e compreender os resultados encontrados em artigos científicos que englobam o uso de recursos fisioterapêuticos no tratamento de estrias presente em mulheres. Compreender a aplicação das técnicas fisioterapêuticas utilizadas na área de dermatofuncional, a fim de garantir e ampliar a gama de ferramentas já existentes em procedimentos estéticos.

\section{Referencial Teórico}

\subsection{Pele}

A pele contém uma membrana flexível e resistente, sendo maior órgão do corpo humano, revestido de heterogêneo e complexo, formada por três camadas de tecidos: Epiderme a camada superior, Derme a camada intermediária e a Hipoderme que a camada mais profunda (Batistela, 2007). Epiderme contém formação de camadas de células sobrepostas, as células superficiais são achatadas e fazem parte da camada córnea rica em queratina, sendo assim a pele tem a classificação de epitélio estratificado pavimentoso queratinizado. A espessura varia dependendo do local do corpo, chegando a 1,5mm nas plantas dos pés. (Borges, 2010).

A derme é localizada abaixo da epiderme, são encontrados vasos sanguíneos, glândulas sebáceas e nervos. Na derme também há tecido subcutâneo, com tecido fibroso, elásticos e gordurosos. As macromoléculas produzidas pelas células mesenquimais e que são envolvidas na composição da derme: fibras elásticas, proteoglicanas e colágeno. (Silva, 2010). A hipoderme tem a função de fixar a epiderme e a derme, sendo a tela subcutânea. O que contribuem para uma reserva de energia no tecido adiposo e a gordura distribuída de forma não uniforme por toda região do corpo. Existem locais que não acumulam gordura, como por exemplo: pálpebras, cicatriz umbilical, pênis e dobras articulares. Mas também existem regiões que têm o maior acúmulo de tecido adiposo como: parede abdominal, porção proximal dos membros e porções laterais (Guirro e Guirro, 2002). 


\subsection{Fisiologia da estria}

A estria pode ser encontrada no sexo masculino ou feminino, sendo que acomete 2,5 vezes em mulheres. Adolescentes do sexo feminino que tenham a idade de doze a quatorze anos e em adolescentes do sexo masculino de doze e quinze anos. Acomete com frequência em obesos, gestantes e pessoas usuários de esteróides (Guirro \& Guirro, 2007).

As estrias são classificadas pelas estruturas com aspectos atróficos e linear, com a região geralmente enrugada, levando a ter alterações cutâneas com trajeto linear, facilmente visível e paralela umas às outras. Inicialmente apresenta uma coloração avermelhada, ao decorrer da evolução do edema causando um processo inflamatório podendo levar a uma coloração esbranquiçada após alguns meses, que pode surgir com pouca quantidade ou numerosas. (Costa,2013) De acordo com coloração inicialmente apresentam com a pigmentação avermelhada com presença de linfócitos, monócitos e neutrófilos ao redor dos vasos sanguíneos, após um tempo onde ocorre a diminuição das fibras de colágeno que evoluem para a coloração branca, denominadas estrias albas (Saito, Zuttin,2014).

As estrias que mudam de cor têm a tendência de ficarem mais profundas, com aparência envelhecida, mais largas e compridas. Se dá por exemplo na obesidade que as estruturas que suportam a pressão geram grandes tensões sobre a pele, assim causando uma lesão e rompimento das fibras elásticas (Guirro \& Guirro,2004). Hoje em dia as estrias são um desafio terapêutico, pelo fato de não existir um tratamento que demonstre a certeza de um resultado fidedigno da melhora completa da pele. $\mathrm{O}$ resultado é variável do tipo de pessoa e pela fase da estria (Kede \& Sabatovich, 2015). As estrias atróficas, são consideradas como sequelas irreversíveis. Alguns autores se baseiam no fato de que não há regeneração da fibra elástica, que é causada por uma má aceitação dos tratamentos para as mesmas (Guirro \& Guirro, 2004).

\subsection{Processo inflamatório da pele}

O processo inflamatório tem a principal função de destruir ou reduzir o agente agressor, que em seguida tem a função de estabelecer uma cura e organização do tecido lesionado. Geralmente essa fase se dá por início no momento de uma lesão, que levará um sangramento que estimula as plaquetas, hemácias e fibrina, dando início ao processo de cicatrização (Borges,2010). $\mathrm{Na}$ fase proliferativa ocorre o processo de migração de fibroblasto que é auxiliada pela citocinas na formação da ferida no processo de crescimento. Ocorrerá nova formação de vasos sanguíneo, angiogenesis irá ajudar na formação de nova matriz, as células ao redor da ferida serão estimuladas ao crescimento. Quando há crescimento de filiforme surgirá uma nova membrana basal próximo a ferida fechando a epiderme (Kede \& Sabatovich, 2015).

A última fase é a da remodelagem na qual o colágeno do tipo 3 passara com tempo no processo de quebra para ser transformado para o tipo1, terá um aumento de ação de fibroblasto, a cicatriz vai se desenvolver para uma cicatriz resistente com organização das fibras (Guirro \& Guirro, 2004). Os contribuintes para o crescimento são proteínas reguladoras, mediadores biológicos que auxiliam no processo de reparo e regeneração celular. Sendo encontrado em tecidos na fase de cicatrização ou renovação da pele (Kede \& Sabatovich, 2015).

\subsection{Microgalvanopuntura}

A técnica de microgalvanopuntura se baseia em gerar estímulos elétricos causado pela corrente galvânica, esse estímulo irá ocasionar modificações eletroquímico nas proximidades da agulha que facilitará alcançar o ponto elétrico de algumas proteínas orgânicas. A corrente galvânica irá desencadear um processo inflamatório agudo na região aplicada, o que levará o tempo de intensidade e duração da reação vai depender da intensidade da corrente como pela capacidade de sensibilidade do paciente (White, et al. 2008) (Guirro \& Guirro, 2004). 
O estímulo ocasionado através da agulha irá promover um processo de reparação com objetivo de restaurar a integridade dos tecidos. A faixa de 70 a 100 micro ampéres (UA) é o indicado para tratamento de estrias, podendo haver variações dependendo da sensibilidade do paciente (Guirro \& Guirro, 2002).

Após o procedimento, a epiderme ficará mais espessa, terá o aumento de fibroblasto, colágenos, fibras elásticas e aumento da vascularização. Para obter um resultado relevante é necessário ter uma boa avaliação, tamanho e espessura da estria e não realizar o procedimento em pacientes que contém contra indicação (Guirro \& Guirro, 2002).

\subsection{Vacuoterapia}

Vacuoterapia é uma técnica que promove sucção da pele até que ocorra micro lesões, que terá como objetivo de melhorar a circulação sanguínea e troca gasosa na região aplicada (Guirro e Guirro, 2004). A vacuoterapia apresenta resultados significativos na melhora da elasticidade da pele, regeneração do tecido, cicatrizes atróficas e fibróticas (Oliveira, 2016). Ocorrerá uma vasodilatação, ocasionando um pequeno edema que fará um aumento da pressão osmótica, facilitando a permeabilidade capilar, será ativado intercâmbio gasoso entre os tecidos capilares e o líquido extracelular que vai ser drenado para gerar a nutrição celular e troca metabólicas e melhorando a flexibilidade tissular (Borges,2010).

Pela aplicação dessa técnica existem algumas contra indicações como aplicação da vacuoterapia em pessoas diabéticas que terá dificuldades de realizar o processo de cicatrização. Pessoas com câncer são contra indicado pelo fato que o estímulo da vacuoterapia no sistema linfático poderá espalhar as células tumorais. (Santos, et al. 2020).

\section{Metodologia}

A presente revisão sistemática buscou seguir as normativas da declaração PRISMA.

\section{Estratégia de investigação}

O presente estudo buscou esclarecer o seguinte problema norteador: $\mathrm{O}$ uso de recursos fisioterapêuticos tais como, Vacuoterapia e Microgalnopuntura evidencia efeitos positivos no tratamento direto nas estrias?

A busca sistemática dos estudos foi coletada através das plataformas digitais: Google acadêmico, Scielo, Capes Periodica. Para tanto foram utilizados os seguintes descritores combinados: Streae, Wind Therapy, Eletrolifting e Vacuo Therapy. A pesquisa obteve duração de agosto até novembro de 2021.

\section{Critérios de elegibilidade}

A presente revisão sistemática contou com a integração de estudos que atenderam os critérios de inclusão e não se encaixaram nos critérios de exclusão. Os critérios de inclusão: artigos publicados com duração de 10 anos (2012 - 2021), estudos com característica de ensaio clínico, escritos na língua portuguesa, na qual abordassem o enfoque no uso dos recursos fisioterapêuticos vacuoterapia e microgalnopuntura no tratamento de estrias. Estudos avaliados na escala Pedro de acordo com as respectivas diretrizes que determinam a qualidade do estudo sistemático. Os critérios de exclusão: artigos de revisão da literatura, estudos de casos sem evidências plausíveis.

\section{Desfechos de interesse}

Os desfechos de interesse dos estudos selecionados teve base nos seguintes itens: autores do artigos, ano de publicação, as amostras utilizadas, correlação do tempo e frequência do tratamento, variáveis estudadas, e a pontuação na escala PEDro. 


\section{Avaliação da qualidade de estudos}

Após a pesquisa e seleção dos estudos, os mesmos foram avaliados por três pesquisadores de maneira independente. A escala de qualidade PEDro é caracterizada por ser uma ferramenta que avalia os estudos clínicos, na área de ciências humanas com foco na reabilitação. A escala é constituída por 11 itens avaliativos, no qual o item n ${ }^{\circ} 1$ é uma exceção por ser atribuído para todos os estudos que apresentam a elegibilidade referente a descrição da amostra no estudo, desta forma temos 10 itens avaliativos. Os itens 2-9 da escala são destinados a validação de característica interna dos estudos, enquanto os critérios 10-11 avaliam com base nas estatísticas, de uma forma clara e fácil interpretação dos resultados encontrados.

Nesta revisão foi adotado as seguintes faixas de pontuação numérica da escala PEDro: 6-10 considerado de alta qualidade; 4-5 média qualidade; 0-3 baixa qualidade. Em caso de qualquer divergência entre os pesquisadores referente ao resultado de qualidade dos estudos, foi resolvido por meio de discussão.

\section{Resultados}

Durante a busca no banco de dados CAPES PERIÓDICA foram encontrados 6 artigos, enquanto que na base de dados GOOGLE ACADÊMICO foram encontrados 14 artigos, na base de dados SCIELO foram encontrados apenas 1 artigo, assim totalizando 21 estudos. Dentre os 6 artigos encontrados na plataforma CAPES PERIÓDICA 5 foram excluídos pelos seguintes motivos: 4 não abordavam a temática proposta em questão, 1 não possuía amostra randomizada. Dentre os 14 artigos encontrados na plataforma GOOGLE ACADÊMICO 12 foram excluídos os seguintes motivos: 6 não abordavam a temática em questão, 4 por tratar-se de artigo de revisão, 1 por ser de artigo de protocolo, 1 artigo referir-se a análise bibliográfica. $\mathrm{O}$ único artigo encontrado na plataforma de dados SCIELO foi excluído devido não abordar a temática proposta. Portanto, para o presente estudo foram localizados 2 ensaios clínicos randomizados controlados na base de dados GOOGLE ACADÊMICO e 1 na base de dados CAPES PERIÓDICA. Desta forma para compor o presente estudo foram utilizados um total de 3 artigos. A Figura 1 apresenta o fluxograma referente à etapa de seleção dos artigos para o estudo. 
Figura 1 - Fluxograma com informações referente ao processo de seleção dos ensaios clínicos com base nos critérios de elegibilidade.

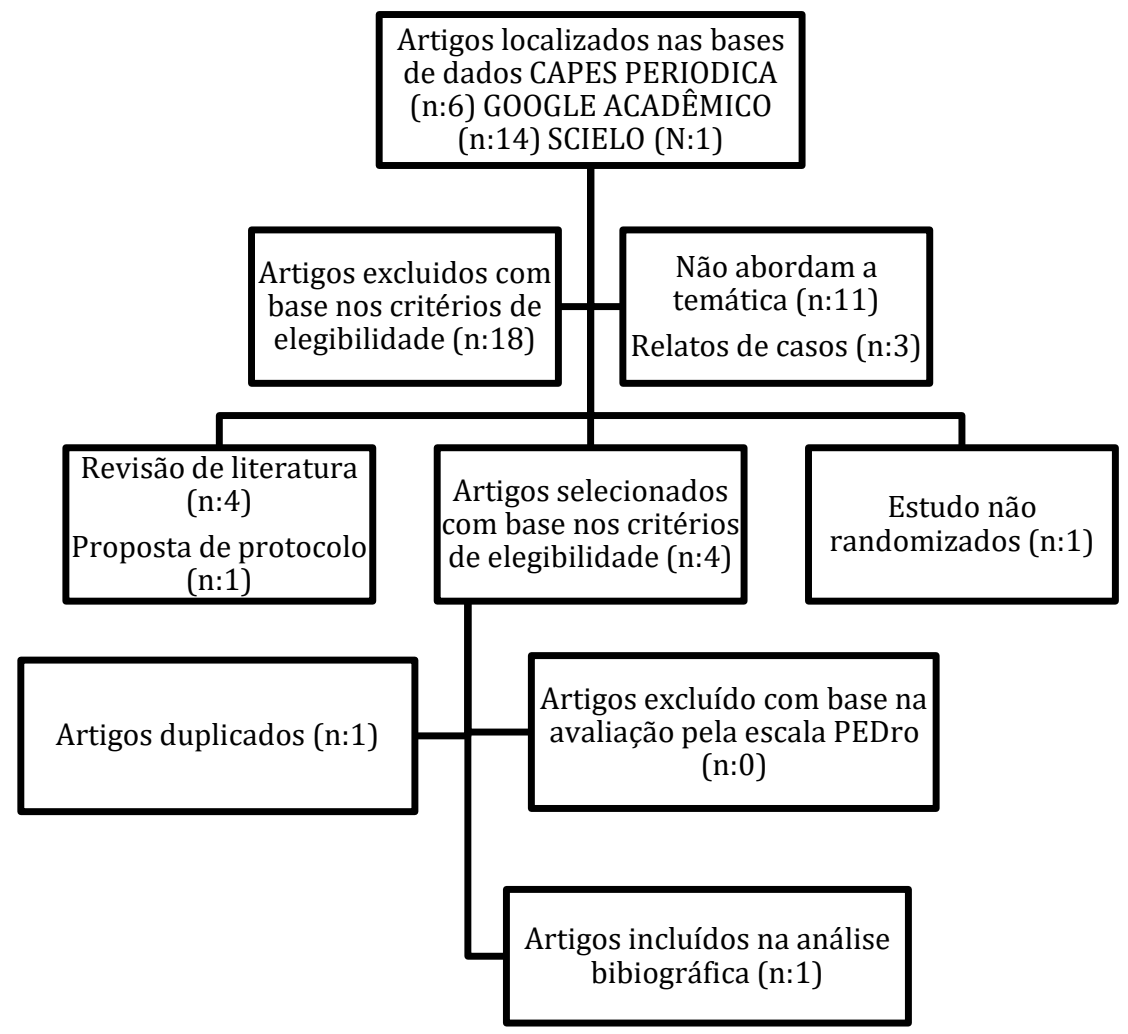

Fonte: Autores.

Todos os estudos selecionados possuíam características de ensaios clínicos com amostras randomizadas, apenas 1 apresentava um grupo controle, na disposição das amostras a predominância maior foi do gênero feminino com a presença de estrias no corpo, que usasse algum recurso fisioterapêutico como abordagem principal de tratamento, todos os estudos obtiveram resultados satisfatórios, ou seja, valores acima de 6 de acordo com a avaliação feita pela escala de qualidade PEDro.

No estudo a amostra mínima dos estudos foi de 01 participante e a máxima foi de 18 participantes, a duração do tratamento variou de 1 mês à 4 meses, com uma frequência de 1x por semana. Os estudos evidenciaram resultados semelhantes entre si, sendo esses: melhora do aspecto da pele tratada, diminuição da espessura das estrias, reparação tecidual e melhora da sensibilidade local. Dentre os estudos $(85 \%)$ apresentou na metodologia o uso de dois grupos paralelos para intervenção, e apenas (15\%) fez uso de grupos independentes para realização das intervenções. Na análise estatística de todos os estudos, o método adotado pelos autores foi de caráter descritivo. Na Tabela 1 observam-se algumas características dos estudos clínicos. 
Tabela 1 - Caracterização dos estudos selecionados.

\begin{tabular}{|c|c|c|c|c|c|}
\hline Estudos & $\begin{array}{l}\text { Amostra (n) } \\
\text { tratamento }\end{array}$ & $\begin{array}{l}\text { Tempo } \\
\text { semanal }\end{array}$ & $\begin{array}{l}\text { Frequência } \\
\text { avaliadas }\end{array}$ & Variáveis & $\begin{array}{l}\text { Pontuação } \\
\text { escala PEDro }\end{array}$ \\
\hline OLIVEIRA & $G V:(1)$ & 1 mês & 1x semana & $\begin{array}{l}\text { Diminuição da } \\
\text { espessura e aspecto } \\
\text { da pele. }\end{array}$ & 6 \\
\hline $\begin{array}{l}\text { FIGUEREIDO } \\
\text { (Et.al) }\end{array}$ & GE: (18) & 2 meses & $\begin{array}{l}1 \mathrm{x} \text { na semana } \\
\text { intervalos } 7 \text { dias }\end{array}$ & $\begin{array}{l}\text { Regeneração } \\
\text { tecidual, aspecto da } \\
\text { pele. }\end{array}$ & 6 \\
\hline ALVES & GE: (1) & $\begin{array}{l}4 \text { meses e } 2 \\
\text { semanas }\end{array}$ & 1x semana & $\begin{array}{l}\text { Reparação } \\
\text { tecido, melhora da } \\
\text { sensibilidade, } \\
\text { neovascularização } \\
\text { local. }\end{array}$ & 8 \\
\hline
\end{tabular}

Fonte: Autores.

$\mathrm{Na}$ avaliação da qualidade metodológica a pontuação média alcançada de acordo com o uso da escala de qualidade PEDro foi de 6, na qual a pontuação mínima foi 6 e máxima de 8. Não foi observado nenhuma discrepância na análise dos dados fornecidos por esses pesquisadores. Uma visão detalhada dos estudos selecionados para esta revisão é bem descrita logo abaixo na Tabela 2 .

Tabela 2 - Pontuação na escala PEDro para estudos selecionados.

\begin{tabular}{|c|c|c|c|c|c|c|c|c|c|c|c|}
\hline Estudos & 1 & 2 & 3 & 4 & 5 & 6 & 7 & 8 & 9 & 10 & 11 \\
\hline OLIVEIRA & $\mathrm{S}$ & $\mathrm{N}$ & $S$ & $S$ & $\mathrm{~N}$ & $\mathrm{~N}$ & $\mathrm{~N}$ & $\mathrm{~S}$ & $\mathrm{~S}$ & S & S \\
\hline ALVES & $\mathrm{S}$ & $\mathrm{N}$ & $\mathrm{N}$ & S & $\mathrm{N}$ & $\mathrm{N}$ & $S$ & $\mathrm{~N}$ & $\mathrm{~S}$ & $S$ & $\mathrm{~S}$ \\
\hline FIGUEREDO & $\mathrm{S}$ & $\mathrm{S}$ & $\mathrm{N}$ & $\mathrm{S}$ & $\mathrm{S}$ & $\mathrm{N}$ & $\mathrm{S}$ & $\mathrm{N}$ & $\mathrm{S}$ & $\mathrm{S}$ & $\mathrm{S}$ \\
\hline
\end{tabular}

(2) = Alocação randomizada; (3) = Atribuição mascarada; (4) = Similaridade no início do tratamento; (5)= assuntos cegos; (6) = Fisioterapeutas cegos; $(7)$ = avaliadores cegos; $(8)=$ acompanhamento apropriado; $(9)$ = análise por intuito de tratar; $(10)=$ correlações intergrupos; $(11)=$ uso de medidas de precisão e variabilidade. Fonte: Autores.

\section{Discussão}

De acordo com Oliveira (2016), em seu estudo clínico foi possível analisar e mensurar os resultados da aplicação do recurso terapêutico denominado vacuoterapia em indivíduos do sexo feminino, que apresentaram desenvolvimento de estrias em áreas do corpo. A maior predominância das estrias são em mulheres na qual apresentam uma taxa de acometimento em média de 2,5 vezes comparado com o sexo masculino que tem uma predisposição menor de apresentar estrias nas áreas corporais. O processo de formação de estrias pode ter uma predisposição maior caso o indivíduo seja obeso, período gestacional e faça uso de medicamentos esteroidais (Guirro \& Guirro, 2007).

Segundo Costa (2013), as estrias são classificadas pelas estruturas com aspectos atróficos e linear, com a região geralmente enrugada, levando a ter alterações cutâneas com trajeto linear, facilmente visível e paralelas umas às outras. Inicialmente apresenta uma coloração avermelhada, ao decorrer da evolução do edema causando um processo inflamatório podendo levar a uma coloração esbranquiçada após alguns meses, que pode surgir com pouca quantidade ou numerosas. Por este motivo a pesquisadora Oliveira, 2016, escolheu como recurso terapêutico a vacuoterapia, por caracterizar-se por um tratamento não invasivo, que age diretamente na estrutura da pele, gerando um desfibrosamento, um aumento da produção de substâncias 
essênciais para o reparo tecidual sendo esses: fibroblastos, colágeno e elastinas, além de uma hipervascularização da área correspondente ao procedimento.

Neste presente estudo, é possível notar o cuidado que a respectiva pesquisadora teve durante a aplicação da técnica de vacuoterapia, que são os parâmetros adequados, desta forma a pressão exercida pelo aparelho deve ser em média entre os valores de 400 a $500 \mathrm{mmHg}$, capazes de atingir um bom resultado clínico (Borges, 2010). Além disso, também vale ressaltar as contra indicações da vacuoterapia, como em indivíduos com diabetes, que irá apresentar dificuldade no processo de cicatrização, indivíduos são contra indicados pelo fato da técnica ocasionar o estímulo no sistema linfático, assim proporcionando o processo de metástase no corpo (Santos, et al. 2020).

Durante uma pesquisa de tratamento de estrias foram selecionadas 20 mulheres com estrias nos locais de coxa, lateral da pelve e glúteo. Foi aplicada nas estrias a técnica de microgalvanopuntura com total de 20 sessões em estrias albas e rubras, ambas obtiveram resultado positivo com melhora de aspecto da pele e diminuição de até 1 centímetro. De acordo com o autor o uso da técnica de microgalvanopuntura teve o aumento do fibroblasto no local, fibras de colágeno e elástica. Também pode-se observar uma melhora de vascularização e sensibilidade no local tratada. (White, 2007).

Em outra pesquisa de estudo acerca do tratamento de estrias, foi realizado aplicação de duas técnicas para poder avaliar a qualidade dos resultados encontrado nas estrias, foram selecionadas no total de 22 pacientes sendo que 11 mulheres receberam o tratamento com a microgalvanopuntura e as outras 11 receberam um tratamento placebo com apenas aplicação da a punturação. O total de sessão variou de acordo com local aplicado na paciente, durante o tratamento com a microgalvanopuntura alguns pacientes relataram melhorar no aspecto da aparência das estrias. Em relação às paciente que receberam aplicação de punturação transversal e perpendicular também tiveram respostas positivas, no final do tratamento foi concluindo que as duas técnicas obtiveram melhoras no tratamento de estrias em relação aparência e diminuição das estrias (Figueiredo, Moura, Machado. 2014).

A corrente galvânica tem como objetivo de causar um processo inflamatório na região aplicada, tendo como estimulado através da agulha aplicada no local. Esse processo levará a uma reparação dos tecidos afetados. (Guirro e Guirro, 2002). Essa técnica tem o benefício de estimulação sensorial, vascularização, nutrição do local afetado, melhora no processo de cicatrização através dos efeitos inflamatório, causado pelo estímulo da puntura da agulha, sendo o principal meio, o qual a corrente penetrará pela pele na estria (White, 2007).

Alguns fatores são contra indicados para tratamento com a corrente galvânica devido à eletricidade aplicada, tais como níveis elevados de glicocorticóides, síndrome de Cushing ou na puberdade, após a gravidez, devido às alterações hormonais, portadores de doenças cardíacas, de neoplasias, de vitiligo, gestantes, epiléticos ou qualquer outra doença que não poderá receber estímulos elétrico (Bravim, 2007).

\section{Conclusão}

De acordo com os estudos encontrados foi possível observar de modo geral, que o uso da vacuoterapia e microgalvanopuntura denominados como recursos fisioterapêuticos, são ferramentas essenciais para tratamentos em pacientes com estrias. Ambas as técnicas tem como objetivo proporcionar a estimulação da produção de fibroblastos e colágeno, melhorar o aspecto da aparência da estria, melhorar a vascularização da área tratada. As técnicas aplicadas tiveram resultados positivos por meio de aplicação de maneira padronizada ou associada com outros recursos que auxilie e potencializem os resultados esperados. Sendo assim sugere-se que na aplicação ambos podem-se optar pelo tratamento não invasivo no caso de vacuoterapia ou no procedimento invasivo com a utilização de agulhas como a microgalvanopuntura. É necessário analisar a situação de cada paciente, antes de qualquer procedimento realizado com os recursos citados acima, desta maneira favorecendo melhor conforto ao paciente. 


\section{Referências}

Almeida, A., Diniz, J., Oliveira, N. \& Torres, R. (2009). Estudo comparativo do tratamento de estrias atróficas com microcorrente galvânica utilizando as técnicas de escarificação e ponturação. p.13. https://docplayer.com.br/21168578-Ana-luiza-o-c-de-almeida1-julia-souki-diniz2-nubia-f-c-oliveira1-rosa-m-dtorres1.html

Araújo, A. M. F. \& Moreno, A. M. (2003). tratamento fisioterápico dermato-funcional por estimulação das estrias com corrente galvânica filtrada. fisio \& terapia v.7. p.31-33

Bizarrias, R., Raposo, W., Péres, M. (2017). Eficácia da eletrogalvanopuntura comparado à acupuntura na técnica de pica-pau (sangria) no tratamento de estrias nacaradas em mulheres pós-gravidez. Fisioter Bras v. 4 p. 489-496

Borges, F. S. (2006). Dermato funcional: Modalidades terapêuticas nas disfunções estéticas. Ed. Phorte.

Borges, M. L. S., Freitas, P. V., Furtado, R. M., Rodrigues, R. B. (2007). Comparação entre o striat e o tratamento com acupuntura em estrias pardas na região de flancos - um estudo de caso. www.portalunisaude.com.br

Carneiro, P. F. C., Santis, S. A. C. (2017). Os principais procedimentos estéticos utilizados no tratamento das estrias.

Calogeno, R. (2017). Tipos de utilização da ventosaterapia. Faculdade De Tecnologia EBRAMEC. P.53,

Figueiredo,S., Moura, S., Machado, D. (2014). Utilização da galvanoterapia na regeneração de estrias atróficas: um estudo piloto sobre a satisfação com o tratamento. Fisioterapia Brasil - V. 15, p. 3

Lopes R. D. S., Vieira C. L. J., Trajano E. T. L.(2015) Aplicação da microcorrente galvânica no tratamento das estrias rubras pósgestação: relato de caso. Revista de Saúde. V.2 p.31-34.

Oliveira, I. (2016). Análise dos efeitos provocados pela utilização da vacuoterapia associada á aplicação da vitamina c nas estrias brancas: um relato de caso. v.1 p. $1-45$

Ponte, M. G. (2011). Recursos fisioterapêuticos utilizados no tratamento das estrias: uma revisão de literatura. Trabalho de pós-graduação em Fisioterapia Dermato-funcional - Faculdade Ávila, p. 1-15 https://docplayer.com.br/22860042-Recursos-fisioterapeuticos-utilizados-no-tratamento-das-estrias-uma-revisaode-literatura.html

Queiroz, J. F. F. Associação dos efeitos do microdermoabrasão e o eletrolifting nas estrias nacaradas. Juazeiro Do Norte-CE: Centro Universitário Dr. Leão Sampaio. V. 1 p.1-36

Rêgo, A., Soares, B., Oliveira, H., Mendes, C., Rocha, L. (2021). Influência da micropuntura associada à micropigmentação no tratamento de estrias albas. Fisioter Bras. V.22 p.1-12

Silveira, C. F. C. (2016). Utilização de corrente microgalvânica para o desenvolvimento de dois protocolos para tratamento de estrias albas. Dissertação Programa de Pós-Graduação em Engenharia Biomédica, Universidade Tecnológica Federal do Paraná. Curitiba. v.1 p. 1-107

Silva, L. F. (2013). Recursos fisioterapêuticos utilizados no tratamento das estrias. Artigo apresentado ao curso de Especialização em Fisioterapia DermatoFuncional do Centro de Estudos Avançados e Formação Integrada, Pontifícia Universidade Católica de Goiás. Goiânia. v.1 p.1-15

Silva, E., Penha, F., Paranhos, I., Bernardes, M., Figueiredo, S. (2020). terapia combinada para tratamento das estrias pós puerpério: benefícios da radiofrequência, vacuoterapia e fatores de crescimento. Brazilian Journal of Natural Sciences V.3, p. 1-9

Reis, C. (2018). Recursos terapêuticos no tratamento de estrias. Revista Saúde Integrada, v. 11 p.1-12

Meyer, P., Moraiws, F., Lima, D., Ronzio, O., Carvalho, M. (2009). Aplicação da galvanoterapia em uma máquina de tatuar para tratamento de estrias. Fisioterapia Brasil - Volume 10 p.1-5

Moreira, J., Giusti, H. (2013). A fisioterapia dermato - funcional no tratamento de estrias: revisão da literatura. v. 1, p. 1-11

Galdino, A., Dias, K. (2010). Análise comparativa do efeito da corrente microgalvânica: estudo de caso no tratamento de estrias atróficas. Revista Eletrônica Saúde CESUC, Volume 10, p. 1-10

Guirro, E. C. O., Guirro, R. R. J. (2004). Fisioterapia dermato-funcional: fundamentos, recursos e patologias. $3^{\text {a }}$ Ed. Rev. E ampliada. Barueri, SP: Ed. Manole.

Guirro, E., Guirro, R. (2002). Fisioterapia Dermato Funcional, 3ed. São Paulo: Manole.

Guirro, E., Guirro, R. (2007). Fisioterapia Dermato-funcional. 3. Ed. Ver.E amp. Sao Paulo: Manole.

Junior, A., Silva, R., Silva, V., Paulino, E. (2018). Estrias: fisiopatologia, principais tratamentos estéticos. P. 1-26. https://semanaacademica.org.br/system/files/artigos/estrias_fisiopatologia_principais_tratamentos_esteticos.pdf

White, P. A. S., Gomes, R. C., Mendonça, A. C., Braganholo, L. D. P., Ferreira, A. S. (2008). Efeitos da galvanopuntura no tratamento de estrias atróficas. Fisioterapia Brasil. v. 9, p. 1-11 